\title{
The Acoustics of Mangetti Dune !Xung Clicks
}

\author{
Amanda Miller ${ }^{1}$ and Sheena Shah ${ }^{2}$ \\ ${ }^{1}$ Department of Linguistics, University of British Columbia, Vancouver, BC, Canada \\ 2 Department of Linguistics, Georgetown University, Washington, D.C., U.S.A. \\ amandamieinterchange.ubc.ca, ss723@georgetown.edu
}

\begin{abstract}
We document the acoustics of the four Mangetti Dune !Xung coronal clicks. We report the temporal measures of burst duration, relative burst amplitude and rise time, as well as the spectral value of center of gravity in the click bursts. COG correlates with lingual cavity volume. We show that there is inter-speaker variation in the acoustics of the palatal click, which we expect to correlate with a difference in the anterior constriction release dynamics. We show that burst duration, amplitude and rise time are correlated, similar to the correlation found between rise time and frication duration in affricates.

Index Terms: clicks, stop acoustics, lingual airstream, rise time, affricates

\section{Introduction}

Mangetti Dune !Xung, henceforth M. D. !Xung, is a member of the $\mathrm{Ju}$ branch of the $\mathrm{Ju}-\neq$ Hoan language family spoken in Mangetti Dune, Namibia. !Xung spoken in another region of Northern Namibia [1] has been described as having the four coronal clicks described by the International Phonetic Association [2]. The full range of M. D. !Xung clicks have not been documented phonetically. Miller-Ockhuizen and Sands [3] showed that M. D. !Xung contains a set of lateral clicks in words that contain alveolar clicks in the related language $\mathrm{Ju}$ 'hoansi. Initial investigations of the acoustics of M. D. !Xung lateral clicks as produced by one female speaker found a fifth click type, which they describe as a forward released dental lateral click, and transcribe as [I||] [3]. In our fieldwork, we found only one type of lateral click in M. D. !Xung, even for the same speaker who participated in the earlier study. The four coronal clicks in M. D. !Xung are provided in Table 1.
\end{abstract}

\begin{tabular}{|c|c|c|c|}
\hline Dental & $\begin{array}{c}\text { Central } \\
\text { Alveolar }\end{array}$ & $\begin{array}{c}\text { Lateral } \\
\text { Alveolar }\end{array}$ & Palatal \\
\hline 1 & $!$ & $\|$ & $\ddagger$ \\
\hline
\end{tabular}

Table 1. Mangetti Dune !Xung click contrasts

This paper reports on the results of an acoustic investigation of all four clicks found in M. D. !Xung for four speakers ( 2 female and 2 male speakers). This study is part of a larger documentation project on M. D. !Xung. We compare the acoustic results to published acoustic records available for !Xóõ in [4], Ju|'hoansi in [5], Naro in [6], IsiXhosa in [7] and $\mathrm{N} / \mathrm{uu}$ in [8] and [9]. Waveforms showing an example of each of the four contrastive M. D. !Xung clicks are provided in Figure 1.

Results show that the dental $[\mid]$ and palatal $[\neq]$ click bursts are shorter in duration than the central alveolar [!] and lateral alveolar [ll] clicks. The palatal click burst duration displays inter-speaker variation. For some speakers it is similar in duration to the dental click, reflecting a noisier release, while for other speakers it is shorter in duration. We expect that the durational differences are due to differences in release dynamics in the articulation. All of the click bursts are about equal in amplitude to the beginning of a following vowel. The dental click is the lowest in amplitude among the four clicks. We show that rise time and burst amplitude display a linear relationship, while rise time and burst duration largely display an exponential relationship. The center of gravity $(\mathrm{COG})$ of the bursts is highest for the dental click, followed by the palatal click and the lateral click, with the alveolar click being the lowest. The COG results directly reflect the lingual cavity volumes found for these clicks by one of the speakers (JF) reported in Miller, Scott, Sands and Shah [10].

(a)

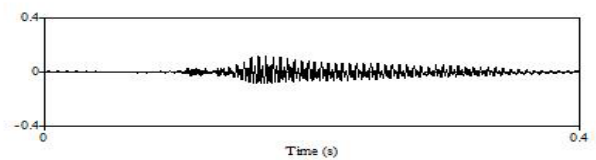

(b)

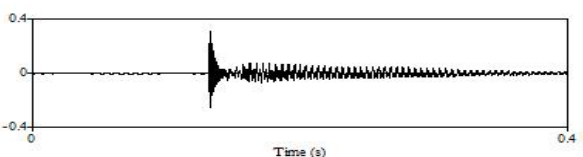

(c)

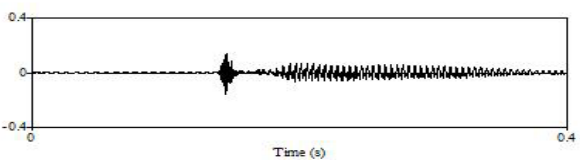

(d)

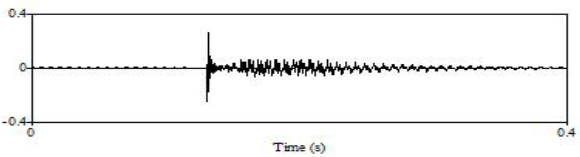

Figure 1: Waveforms of the four coronal clicks in Mangetti Dune! Xung in the words (a) ${ }^{\mathrm{g}}$ |uu (dental click), (b) ${ }^{\mathrm{g}}$ !uu (central alveolar click),(c) ${ }^{\mathrm{g}} \|$ uu (lateral alveolar click) and (d) qłuu (palatal click, speaker JF).

\section{Methods}

We recorded 15 repetitions of the wordlist in (1) with four speakers of M. D. !Xung. Two speakers were male (JF and $\mathrm{MA}$ ), and two were female (BC and TK). All were in their twenties. The words were recorded in the frame sentence $\mathrm{Ya} /$ ha __ y $\left.\right|^{\mathrm{h}} \mathrm{mm}$. 'His/her __ is nice.' The choice of ya / ha as the $\overline{3^{\text {rd }}}$ person pronoun is dialectal, although it was difficult to draw a division between dialects otherwise. Both are gender neutral.

While the clicks recorded here are voiced, this should not affect comparisons with voiceless clicks in other studies, because the voicing is only found in the closure, and there is no voicing during the burst or Voice Onset Time phase.

(1) Wordlist used in this study

g|uu 'refusal to give s.t. to s.o.' g! uu 'belly'

9"uu 'water' 9quu 'tick' 
The phrases were recorded using a Sony ECM-144 electret condenser microphone, a Sound Devices USB Pre combined pre-amp connected to an iBook computer, and were sampled at $44,100 \mathrm{~Hz}$.

Begin closure (bc), begin burst (bb), end burst (eb), begin vowel (bv) and end vowel (ev) labels were marked using Praat (Boersma and Weenink 2009). The 'bc' label was marked at the beginning of voicing. The 'bb' and 'eb' labels were placed at the beginning and the end of the burst respectively. 'bv' was labeled at the beginning of the vowel. The label indicating the end of the vowel was difficult to place because of vowel nasalization due to anticipatory coarticulation. We marked the 'ev' label at the end of F3 in the vowel.

We wrote a Praat script to calculate the click burst duration, the rise time to peak amplitude in the burst described in [4] and [5]; burst amplitude relative to the amplitude at the beginning of the vowel, and the burst center of gravity. The burst amplitude measure calculated the amplitude on the basis of the root mean squared (RMS) amplitude, not the raw waveform, as did the Rise Time calculation.

While burst amplitude is a spectral measure rather than a temporal measure, the results in this paper show that the burst amplitude is highly correlated with the burst duration. It is due to the relatedness of these measures that we include burst amplitude in a section on temporal measures.

\section{Results}

\subsection{Temporal properties}

\subsubsection{Click burst duration}

Figure 2 provides the burst duration of the different clicks for all four speakers. All speakers display a longer burst duration for the dental [9] and lateral alveolar [ $\left.{ }^{9}\right]$ clicks compared with the central alveolar $\left[{ }^{9} !\right]$ and palatal $\left[{ }^{9} \neq\right]$ clicks. The dental click displays a high degree of variability for speaker BC. The burst duration differences are consistent with a previous study of Naro click burst duration in [6]. The duration contrast is part and parcel of a frication contrast that has variously been couched in terms of different manner contrasts, e.g. affricate vs. implosive [12], fricative vs. occlusive in Trubetzkoy [13], and delayed vs. spontaneous release in Chomsky and Halle [14 ]. Johnson [15] focuses on the acoustic property of noisy release, which results from a fricated release for $\left[{ }^{9}\right]$ and a lateral release for [\|]. Ladefoged and Traill [4] classify these two clicks as [+noisy].
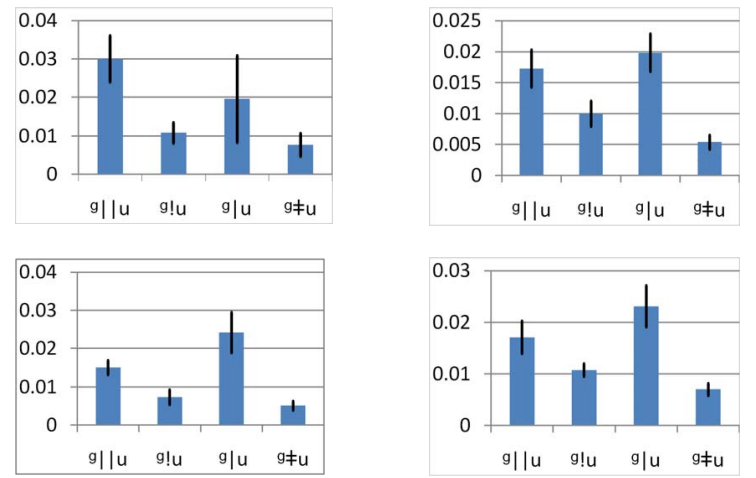

Figure 2: Burst Duration of the four click types in Mangetti Dune !Xung (speakers BC, TK, Upper, speakers $J F, M A$, Lower).

Quantitative results for M. D. !Xung click bursts are similar to those found for !Xóõ [4], but the noisy clicks in
Naro reported in [6] are longer, closer to $30 \mathrm{~ms}$. Speakers TK and MA display bursts that are twice as long for [ ${ }^{9}$ ! compared with [ $\left.{ }^{9} \neq\right]$, similar to that found in !Xóõ [4]. Speakers BC and JF's abrupt clicks have similar durations, which is comparable to the pattern reported for Naro [6] and N|uu [16]. We suspect that the inter-speaker variation found in the M. D. !Xung [ $\left.{ }^{9} \neq\right]$ burst durations may be due to variation in the release dynamics of the [9 $\neq$ ] click for these speakers. Sands [7] and Fulop et al. [17] report total release durations for the IsiXhosa and Siyeyi clicks, which include both the anterior burst and voice onset time, thus the results are not comparable to the other studies.

\subsubsection{Click burst amplitude}

Ladefoged and Traill [4] note that clicks in !Xóõ often have a peak-to-peak voltage ratio that is more than twice that of the onset of the following vowel (about a $6 \mathrm{~dB}$ difference in intensity), and they note that this is an important acoustic feature that distinguishes clicks from other stops. Traill [18] provides an intensity scale as in Table 2, and states that there is a great degree of variability. Miller-Ockhuizen [19] shows similar results for Jul'hoansi, and also comments on the great degree of variability. The $[\odot]$ symbol in Table 2 corresponds to the bilabial click that is not found in M. D. !Xung.

$$
\text { !, } \| \quad \gg \quad \neq>\quad \mid, \odot \quad>\quad \text { t, k, q }
$$

Table 2. Scale of intensity of noise-bursts for !Xóõ consonants (from Traill [18])

Figure 3 reports the relative burst amplitude of the four clicks in M. D. !Xung. As can be seen, the clicks are generally similar in amplitude relative to the midpoint of the vowel. The different clicks display some variation, with the dental click [l] having the lowest amplitude relative to the vowel for all speakers.
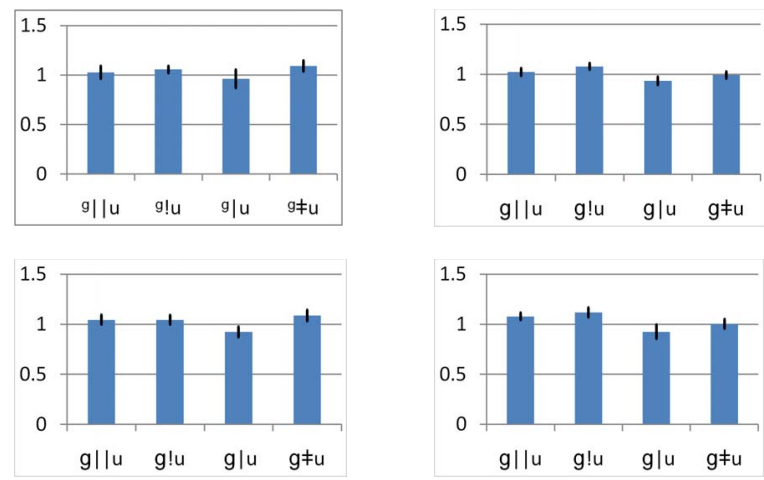

Figure 3: Relative Burst Amplitude of the four click types in Mangetti Dune !Xung (speakers BC, TK, Upper, speakers JF, MA, Lower).

\subsubsection{Rise time}

Ladefoged and Traill [4] report rise time to peak amplitude for clicks, and relate this measure to the abrupt vs. noisy characteristic of click bursts. The rise time measure used here is similar to rise time used to measure affricates in Howell and Rosen [20], except that we are concerned with the rise time to peak amplitude in click bursts rather than in frication noise. Diehl, Molis and Castleman [21] show that rise time is related to frication duration, and that frication 
duration is more perceptually relevant than rise time in English affricates. We thus explore here the relationship between rise time in the click burst, click burst amplitude, and click burst duration in M. D. !Xung.

Figure 4 provides the rise time to peak amplitude plotted against the relative burst amplitude. The results show that a shorter rise time correlates with a higher burst amplitude, and vice versa.
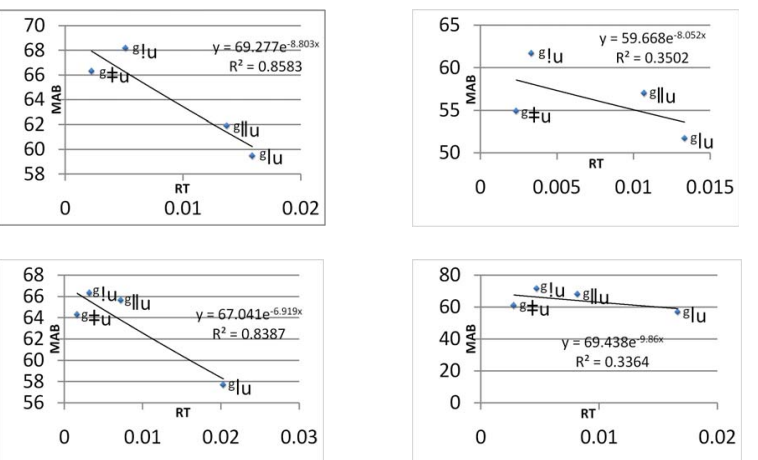

Figure 4: Rise Time (RT) against relative burst amplitude (MBA) of the four click types in Mangetti Dune!Xung (speakers BC, TK, Upper, speakers JF, $M A$, Lower).

Figure 5 shows rise time against burst duration. Rise time increases alongside burst duration. For three of the speakers (BC, TK and MA), the relationship is exponential, while for one of the speakers (JF), the relationship is linear.
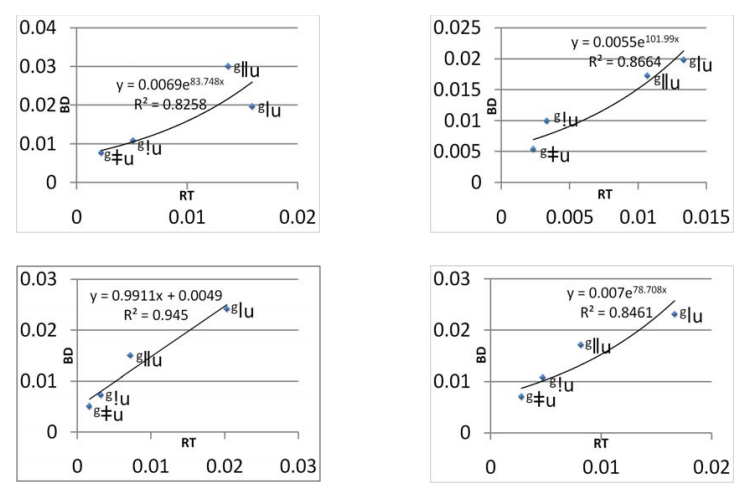

Figure 5: Rise Time (RT) against burst duration (BD) of the four click types in Mangetti Dune !Xung (speakers BC, TK, Upper, speakers $J F, M A$, Lower).

\subsection{Center of Gravity}

Figure 6 provides the center of gravity (COG) results. For three out of four speakers (TK, JF, MA), the dental click has the highest COG (approximately $4000 \mathrm{~Hz}$ for the female speakers, and approximately $3000 \mathrm{~Hz}$ for the male speakers). The palatal $\left[{ }^{9} \neq\right]$ click is also relatively high (2500-3000 Hz for the female speakers, and $1500-2000 \mathrm{~Hz}$ for the male speakers). Female speaker BC does not show a difference between the COG of the $\left[{ }^{9} \mid\right]$ and $\left[{ }^{9} \neq\right]$ clicks. The lateral click has a much lower COG at around 2000 $\mathrm{Hz}$, and the COG of [?!] is the lowest at around $1000 \mathrm{~Hz}$ for all speakers. The M. D. !Xung results are similar to the $\mathrm{N} \mid \mathrm{uu}$ values reported in [16].

\section{Discussion}

\subsection{Temporal properties}

The dental and lateral clicks in M. D. !Xung exhibit longer burst durations, longer rise times to peak amplitude in the burst, and lower amplitudes than are found for the alveolar [?!] and palatal [ $\left.{ }^{9} \neq\right]$ clicks. These properties all arise from the more fricated bursts found in the dental [9 $\left.{ }^{9}\right]$ and lateral [ ["]] clicks. However, there is no reason to view these clicks as affricates, as opposed to noisy stops. Similarly, Chomsky and Halle's [14] characterization of the clicks with delayed vs. spontaneous release is not quite right, because the difference is due to a slower release, rather than a delayed release.
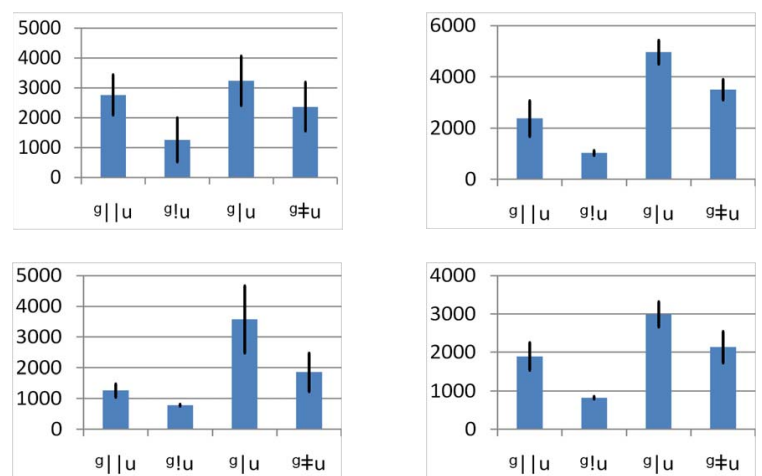

Figure 6: Center of Gravity of the four click types in Mangetti Dune!Xung (speakers BC, TK, Upper, speakers JF, MA, Lower).

Johnson's [15] and Ladefoged and Traill's [4] classification of the clicks in terms of the feature [noisy] captures this acoustic string of properties appropriately. Further, it is applicable to pulmonic stops as well as clicks, e.g. the palatal pulmonic stop which exhibits a high degree of frication noise in many languages.

The strong relationship found between burst duration, rise time and burst amplitude underlies the need for a perception experiment to determine the weighting of these acoustic cues. Since clicks only occur initially in M. D. !Xung and most other non-Bantu click languages, we don't expect there to be much contextual variation (though vowel context may play a role).

We have reported inter-speaker variation in the duration of $\left[{ }^{9} \neq\right]$ within the same language. This study has shown the relationship between burst duration, rise time and amplitude of click bursts. Following Johnson, we have noted that the frication arises from two articulatory sources: lateral articulations, and laminal dental articulations. The alveolar click has been shown to be apical in !Xóõ [23] and N|uu [22]. The palatal click has broad anterior contact patterns in !Xóõ [23] and N|uu [22]. However, Miller et al. [16] note that the broad contact may be due to sliding of the anterior constriction, and that static palatography does not allow release dynamics to be captured. Our palatograms for Mangetti Dune !Xung also display broad contact patterns. We expect that the variants produced by two of the M. D. !Xung speakers may correspond to differences in the anterior constriction release dynamics. High-speed ultrasound studies such as Miller, Scott, Sands and Shah [10] for all of the speakers will contribute more detail about the release dynamics. 


\subsection{Center of Gravity}

The center of gravity results largely match the findings found in previous studies - namely the $\left[{ }^{9}\right]$ and $\left[{ }^{9} \neq\right]$ clicks have higher center of gravities than the [ ${ }^{9}$ ! $]$ click, with the lateral click [ $\left.{ }^{9} \|\right]$ having an intermediate value. This corresponds to the differences in cavity size seen in high-speed ultrasound imaging reported in Miller, Scott, Sands and Shah [10].

\subsection{Forward released lateral click}

Our linguistic fieldwork and the phonetic results reported here show no evidence for a forward released denti-lateral click originally described by Miller-Ockhuizen and Sands [5]. While speaker SR, who originally produced two lateral clicks in the earlier study, did not participate in the acoustic study, it was clear from our fieldwork that she no longer produces this click. We expect that SR's production of a different click in the earlier study was socially motivated by the excitement she saw in our eyes when she produced the click a bit differently. This underlies the strict importance of not accepting new segments into the IPA without first carrying out a full acoustic study with multiple speakers. It also underscores the fragility of situations where fieldworkers are working with one speaker who is isolated in a different language community, as was the case with SR when we worked with her in Tsumkwe, Namibia (in the heart of the Ju|'hoansi community) in 2000.

\section{Conclusions}

M. D. !Xung has been shown to have four contrastive coronal clicks. The dental, central alveolar and lateral alveolar clicks display burst durations similar to other languages. However, the burst durations for the palatal click display interspeaker variation. For some speakers, the palatal click duration is half that of the alveolar click, while for others the durations of these two clicks are quite similar. The variation in burst duration correlates with the variation seen in burst amplitude and rise time. High-speed ultrasound studies will investigate the dynamics of the palatal click [ $\ddagger]$ across speakers. A perception study is needed to determine how burst duration, burst amplitude, and rise time to peak amplitude in the click bursts are weighted with respect to each other.

\section{Acknowledgements}

We would like to thank our speakers, (Jenggu Rooi Fransisko, Martin $\|$ Oshe Aromo, Sikunda |U'i Fly, Bingo Kanaho Costa, Caroline Tumbo Kaleyi, Sabine Towe Riem), as well as Rosa Tina Kalezi who helped with organization of speakers. We would like to express our thanks also to Pastor Herman Oosthuizen and Elna Oosthuizen for use of their church for our recordings. We would also like to acknowledge the help of Bonny Sands who contributed to the larger documentation project on M. D. !Xung, and participated in the fieldwork where we recorded the data for this study. We would like to acknowledge the support of a National Science Foundation Grant, NSF \#BCS-0726200 (PI Amanda Miller) and BCS-0726198 (PI Bonny Sands): "Collaborative Research: Phonetic and Phonological Structures of Post-velar Constrictions in Clicks and Laterals". Any opinions, findings, and conclusions or recommendations expressed in this material are ours and do not necessarily reflect the views of the National Science Foundation.

\section{References}

[1] Heikkinen, T. "Phonology of the !Xũ dialect spoken in Ovamboland and western Kavango". South African Journal of African Languages, 6 (1), 18-28, 1986.
[2] IPA. 2006. The International Phonetic Alphabet (revised to 2005) [chart]. Journal of the International Phonetic Association 36(1), 135.

[3] Miller-Ockhuizen, A. and Sands, B. "!Kung as a Linguistic Construct". Language and Communication, 19(4): 401-413. In B. Joseph [Ed.]. Social, Cultural, and Political Perspectives on Languages in Conflict, 1999.

[4] Ladefoged, P. and Traill, A. "Clicks and their accompaniments". Journal of Phonetics, 22: 33-64, 1994.

[5] Miller-Ockhuizen, A. and Sands, B. "Contrastive Lateral Clicks and Variation in Click Types". Proceedings of ICSLP 2000, Vol. II. Beijing, China. 499-500, 2000.

[6] Kagaya, R. "Soundspectrographic analysis of Naron clicks -A preliminary report". Annual Bulletin of Institute of Logopedics and Phoniatrics, 12, 113-125, 1978.

[7] Sands, B. "Evidence for click features: Acoustic characteristics of Xhosa clicks". UCLA Working Papers in Phonetics 80, 6-37, 1991.

[8] Miller, A., Brugman, J., Sands, B., Exter, M., Namaseb, L. and Collins, C. The sounds of N|uu: Place and airstream contrasts. Working Papers of the Cornell Phonetics Laboratory 16, Ithaca, NY: CLC Publications, 101-160, 2007.

[9] Miller, A., Brugman, J., Sands, B., Namaseb, L., Exter, M. and Collins, C. " Differences in Airstream and Posterior Place of Articulation among N|uu Lingual Stops", In Press, Journal of the International Phonetic Association, 39.2, 2009.

[10] Miller, A., Scott, A., Sands, B. and Shah, S. "Rarefaction gestures and coarticulation in Mangetti Dune !Xung Clicks". in Proceedings of Interspeech 2009, Brighton, U.K, 2009.

[11] Boersma, P. and Weenink, D. Praat: doing phonetics by computer Version 5.1.04. Online: http://www.praat.org/, Accessed April 1, 2009.

[12] Beach, D. M. "The phonetics of the Hottentot language". Cambridge. W. Heffer \& Sons, Ltd. 1938.

[13] Trubetzkoy, N.S. Principles of Phonology, translated by C. Baltaxe, Los Angeles: University of California Press, 1969.

[14] Chomsky, N. and Halle, M. "The Sound pattern of English". Harper and Row. New York, 1968.

[15] Johnson, K. "Acoustic and auditory analyses of Xhosa clicks and pulmonics". UCLA Working Papers in Phonetics 83. 33-45, 1993.

[16] Miller, A.,Brugman, J. and Sands, B. "Acoustic and auditory analyses of N|uu lingual and pulmonic stop bursts. in J. Trouvain and W. Barry [Eds.], Proceedings of the XVIth International Congress of Phonetic Sciences 2007, 769-772, 2007. Online: http://www.icphs2007.de/conference/Papers/1664/1664.pdf

[17] Fulop, S., Ladefoged, P., Liu, F. and Vossen, R. "Yeyi Clicks: Acoustic Description and Analysis". Phonetica 60, 231-260, 2007.

[18] Traill, A. "Linguistic phonetic features for clicks: Articulatory, acoustic and perceptual evidence". in Herbert, R [Ed.], African linguistics at the crossroads: Papers from Kwaluseni. Köln: Rüdiger Köppe, 1997.

[19] Miller-Ockhuizen, A. The phonetics and phonology of gutturals: A case study from Ju|'hoansi. Outstanding Dissertations in Linguistics. New Haven: Routledge, 2003.

[20] Howell, P. and Rosen, S. "Production and perception of rise time in the voiceless affricate / fricative distinction", JASA. 73 (3), 976984, 1983.

[21] Diehl, R.L., Molis, M.R., and Castleman, W.A. "Adaptive design of sound systems: Some auditory considerations". in K. Johnson and E. Hume [Eds], "The Role of Perceptual Phenomena in Phonological Theory”. San Diego: Academic Press.123-139, 2001 .

[22] Sands, B., Brugman, J., Exter, M., Namaseb, L., Miller, A. "Articulatory characteristics of Anterior Click Closures in N|uu", in Trouvain, J. and Barry, W., [Eds.] Proceedings of the $16^{\text {th }}$ International Congress of Phonetic Sciences, Pirrot GmbH, Dudweiler, Germany, 401-404, 2007, Online: http://www.icphs2007.de/

[23] Traill, A. Phonetic and phonological studies of !Xóõ Bushman Quellen zur Khoisan-Forschung 1. Hamburg: Helmut Buske Verlag, 1985. 